Aleksander Maliszewski*, Department of Mathematics, Pedagogical

University, Chodkiewicza 30, 85-064 Bydgoszcz, Poland,

e-mail:wspb05@@pltumk11.bitnet

\title{
SUMS AND PRODUCTS OF QUASI-CONTINUOUS FUNCTIONS
}

\begin{abstract}
In this article two main results are proved. The first one is that each cliquish function $f: \mathbb{R}^{k} \rightarrow \mathbb{R}$ is the sum of two quasi-continuous functions. It is also shown that we can moreover require that the summands preserve points of continuity of $f$, are bounded provided that $f$ is bounded and belong to the same class of Baire as $f$ (if $f$ is Borel measurable). The other main result is that each function $f: \mathbb{R}^{k} \rightarrow \mathbb{R}$ which can be written as the product of finitely many quasi-continuous functions, can be expressed as the product of two quasi-continuous functions, and we can require that the factors belong to the same class of Baire as $f$ (if $f$ is Borel measurable).
\end{abstract}

\section{Introduction}

The notion of quasi-continuity for real functions of several real variables was introduced over sixty years ago by S. Kempisty [8], as a generalization of the notion of continuity, and it has been intensively studied since then. A comprehensive survey on this topic can be found in [12].

It is easy to show that the sum of two quasi-continuous functions need not be quasi-continuous. However, it must be cliquish, as the sum of two cliquish functions is cliquish. In $1985 \mathrm{Z}$. Grande proved that each cliquish function $f$ defined on $\mathbb{R}$ can be expressed as the sum of four quasi-continuous functions, and in the case where $f$ is locally bounded, as the sum of three quasicontinuous functions [5]. This theorem was generalized by E. Strońska [13], [14], for cliquish functions defined on a separable, metrizable, dense in itself, Baire

Key Words: quasi-continuous function, cliquish function, Lebesgue function

Mathematical Reviews subject classification: Primary: 54C08; Secondary 54C30, 28A15, 26A21

Received by the editors November 11, 1994

* Supported by a KBN Research Grant 2114491 01, 1992-94 
space. Later J. Borsík and J. Doboš proved that each cliquish function defined on $\mathbb{R}^{k}$ is the sum of two simply continuous functions, each of which in turn can be written as the sum of two quasi-continuous functions [3]. The latest result in this topic is that each cliquish function defined on a separable metrizable topological space can be written as the sum of three quasi-continuous functions [1].

The story of investigating the family of functions which can be written as the product of quasi-continuous functions is much shorter. T. Natkaniec proved the following theorem [11] in 1990.

Theorem 1.1 A function $f: \mathbb{R} \rightarrow \mathbb{R}$ can be factored into a (finite) product of quasi-continuous functions iff $f$ is cliquish and

$(\star)$ each of the sets $f^{-1}((-\infty, 0)), f^{-1}(0)$, and $f^{-1}((0, \infty))$ is the union of an open set and a nowhere dense set.

However, in his representation of such functions he uses as many as eight (!) quasi-continuous functions. Lately J. Borsík proved that each real cliquish function defined on a separable metrizable topological space which fulfills condition $(\star)$ can be written as the product of three quasi-continuous functions [2]. Moreover, he simplified the condition $(\star)$ to the following form:

The set $f^{-1}(0)$ is the union of an open set and a nowhere dense set.

(It is possible if the domain is a Baire space.)

It is natural to ask which cliquish functions can be expressed as the sum (resp. the product) of two quasi-continuous functions. If the domain is $\mathbb{R}^{k}$, the answer is all (resp. all satisfying condition $(\star))$.

\section{Preliminaries}

First we need some notation. The real line $(-\infty, \infty)$ is denoted by $\mathbb{R}$ and the set of positive integers, by $\mathbb{N}$. Throughout this article, $k$ is a fixed positive integer. The word function means a mapping from $\mathbb{R}^{k}$ into $\mathbb{R}$ unless otherwise explicitly stated. The words measure, summable etc. refer to Lebesgue measure and integral in $\mathbb{R}^{k}$. The Euclidean metric in $\mathbb{R}^{k}$ is denoted by $\varrho$. For every set $A \subset \mathbb{R}^{k}$ let $\operatorname{diam} A$ be its diameter (i.e., $\operatorname{diam} A=\sup \{\varrho(x, y): x, y \in$ $A\})$, int $A$ its interior, cl $A$ its closure, fr $A$ its boundary, $\chi_{A}$ its characteristic function, and $|A|$ its outer Lebesgue measure. A symbol like $\int_{A} f$ will always mean the Lebesgue integral. For any function $f$ we write $\|f\|$ for $\sup \{|f(t)|$ : $\left.t \in \mathbb{R}^{k}\right\}$ ( $f$ need not be bounded) and we denote by $D(f)$ the set of points of discontinuity of $f$. 
The oscillation of a function $f$ on a non-empty set $A \subset \mathbb{R}^{k}$ will be denoted by $\omega(f, A)$ (i.e., $\omega(f, A)=\sup \{|f(x)-f(y)|: x, y \in A\})$. Similarly, the oscillation of a function $f$ at a point $x \in \mathbb{R}^{k}$ will be denoted by $\omega(f, x)$ (i.e., $\left.\omega(f, x)=\lim _{r \rightarrow 0^{+}} \omega\left(f,\left\{y \in \mathbb{R}^{k}: \varrho(x, y)<r\right\}\right)\right)$.

We say that a function $f$ is quasi-continuous (resp. cliquish) at a point $x \in \mathbb{R}^{k}$ if for each $\varepsilon>0$ and each open set $U \ni x$ we can find a non-empty open set $V \subset U$ such that $\omega(f,\{x\} \cup V)<\varepsilon$ (resp. $\omega(f, V)<\varepsilon)$. We say that $f$ is quasi-continuous (cliquish) if it is quasi-continuous (cliquish) at each point $x \in \mathbb{R}^{k}$. Cliquish functions are also known as pointwise discontinuous.

We will use the following well-known (and easy to prove) facts:

- A function $f$ is quasi-continuous at a point $x \in \mathbb{R}^{k}$ iff there exists an open set $H \subset \mathbb{R}^{k}$ such that $x \in \operatorname{cl} H$ and $f \mid(\{x\} \cup H)$ is continuous at $x$.

- A function $f$ is quasi-continuous iff for each $x \in \mathbb{R}^{k}$ there exists a sequence $x_{1}, x_{2}, \ldots \notin D(f)$ such that $x_{n} \rightarrow x$ and $f\left(x_{n}\right) \rightarrow f(x)$.

- The limit of a uniformly convergent sequence of quasi-continuous functions is quasi-continuous.

- Each quasi-continuous function is cliquish.

- A function $f$ is cliquish iff $D(f)$ is of the first category.

The word interval (resp. cube) will always mean non-degenerate compact interval (resp. cube) in $\mathbb{R}^{k}$, i.e., Cartesian product of $k$ non-degenerate compact intervals (resp. compact intervals of equal length) in $\mathbb{R}$. For every interval $I=\left[a_{1}, b_{1}\right] \times \cdots \times\left[a_{k}, b_{k}\right]$ we denote $I^{\circ}=\left[a_{1}, b_{1}\right) \times \cdots \times\left[a_{k}, b_{k}\right)$.

The proof of the following simple technical lemma is left to the reader.

Lemma 2.1 Whenever $A \subset \mathbb{R}^{k}$ is closed there exists a family $\mathcal{J}$ of nonoverlapping cubes such that each $x \notin A$ belongs to the interior of the union of some finite subfamily of $\mathcal{J}$ and $|J| \leq[\varrho(A, J)]^{k}$ for each $J \in \mathcal{J}$.

We say that a sequence of intervals $\left\{I_{n}: n \in \mathbb{N}\right\}$ is o-convergent to a point $x \in \mathbb{R}^{k}$ if $x \in \bigcap_{n \in \mathbb{N}} I_{n}, \lim _{n \rightarrow \infty} \operatorname{diam} I_{n}=0$, and $\limsup _{n \rightarrow \infty}\left(\operatorname{diam} I_{n}\right)^{k} /\left|I_{n}\right|<\infty$. We will write $I_{n} \stackrel{o}{\Rightarrow} x$. (Cf., e.g., [10].)

By an interval function we will mean a mapping from the family of all intervals into $\mathbb{R}$. For an arbitrary interval function $F$ and a point $x \in \mathbb{R}^{k}$ we define

$$
o \text { - } \limsup _{I \Rightarrow x} F(I)=\sup \left\{\limsup _{n \rightarrow \infty} F\left(I_{n}\right): I_{n} \stackrel{o}{\Rightarrow} x\right\}
$$


and

$$
o-\liminf _{I \Rightarrow x} F(I)=\inf \left\{\liminf _{n \rightarrow \infty} F\left(I_{n}\right): I_{n} \stackrel{o}{\Rightarrow} x\right\}
$$

If the two above limits coincide, then we denote their value by $o$ - $\lim _{I \Rightarrow x} F(I)$.

We say that $x \in \mathbb{R}^{k}$ is an o-Lebesgue point of a function $f$ if $f$ is locally summable at $x$ and $o$ - $\lim _{I \Rightarrow x} \int_{I}|f-f(x)| /|I|=0$. We say that $f$ is an $o$-Lebesgue function if each $x \in \mathbb{R}^{k}$ is an o-Lebesgue point of $f$.

The following theorem is proved in [10].

Theorem 2.2 Assume that $A \subset \mathbb{R}^{k}$ is closed, $\mathcal{J}=\left\{J_{n}: n \in \mathbb{N}\right\}$ is a family of non-overlapping cubes and $\left\{f_{n}: n \in \mathbb{N}\right\}$ is a family of summable functions such that the following conditions are satisfied:

(i) each $x \notin A$ belongs to the interior of the union of some finite subfamily of $\mathcal{J}$,

(ii) $\left|J_{n}\right| \leq\left[\varrho\left(A, J_{n}\right)\right]^{k}$ for each $n \in \mathbb{N}$,

(iii) $f_{n}(x)=0$ if $x \notin J_{n}, n \in \mathbb{N}$,

(iv) for each $x \in A$ and each $\tau>0$ there exists an $\eta>0$ such that for each $n \in \mathbb{N}$, if $\varrho\left(x, J_{n}\right)<\eta$, then $\int_{J_{n}}\left|f_{n}\right| \leq \tau \cdot\left|J_{n}\right|$.

Put $f=\sum_{n=1}^{\infty} f_{n}$. Then the function $f$ is locally summable and moreover, each $x \in A$ is an o-Lebesgue point of $f$. [10, Proposition 7.5]

\section{Auxiliary Lemmas}

The following lemma is a multidimensional version of Lemma 2 of [7].

Lemma 3.1 Let a set $G \subset \mathbb{R}^{k}$, a function $f$ and an $\varepsilon>0$ be such that $\omega(f, x)<\varepsilon$ for each $x \in G$. Then there is a continuous function $\bar{f}: G \rightarrow \mathbb{R}$ such that $|f-\bar{f}| \leq \varepsilon / 2$ on $G$.

Proof. For each $x \in G$ define $m(x)=\min \left\{f(x), \liminf _{t \rightarrow x} f(t)\right\}$ and $M(x)=\max \left\{f(x), \limsup _{t \rightarrow x} f(t)\right\}$. Then $m$ is lower semicontinuous, $M$ is upper semicontinuous, and $M(x)-m(x)=\omega(f, x)<\varepsilon$ for each $x \in G$. By Theorem 1.N(d) of [4], there is a continuous function $\bar{f}: G \rightarrow \mathbb{R}$ such that

$$
f(x)-\varepsilon / 2 \leq M(x)-\varepsilon / 2 \leq \bar{f}(x) \leq m(x)+\varepsilon / 2 \leq f(x)+\varepsilon / 2
$$

for each $x \in G$, whence $|f-\bar{f}| \leq \varepsilon / 2$ on $G$.

Lemma 3.2 Assume that $A$ is a nowhere dense closed set which contains all points of discontinuity of quasi-continuous functions $h_{1}$ and $h_{2}$. Then there exists a family of non-overlapping cubes $\mathcal{G}=\left\{G_{n}: n \in \mathbb{N}\right\}$ such that 
(i) each $x \notin A$ belongs to the interior of the union of some finite subfamily of $\mathcal{G}$,

(ii) $\left|G_{n}\right| \leq\left[\varrho\left(G_{n}, A\right)\right]^{k}$ for each $n \in \mathbb{N}$,

(iii) for $i \in\{1,2\}$ and each $x \in A$ there exists a subfamily $\left\{G_{n_{l}}: l \in \mathbb{N}\right\}$ such that $x \in \operatorname{cl} \bigcup_{l \in \mathbb{N}} G_{n_{l}}$ and $h_{i} \mid\left(\{x\} \cup \bigcup_{l \in \mathbb{N}} \operatorname{int} G_{n_{l}}\right)$ is continuous at $x$.

Proof. First choose a family $\left\{I_{p}: p \in \mathbb{N}\right\}$ of non-overlapping cubes according to Lemma 2.1. For each $p \in \mathbb{N}, h_{1}$ and $h_{2}$ are uniformly continuous on $I_{p}$, so we can find non-overlapping cubes $G_{p, 1}, \ldots, G_{p, l_{p}}$ such that $I_{p}=\bigcup_{j=1}^{l_{p}} G_{p, j}$ and $\omega\left(h_{i}, G_{p, j}\right)<\varrho\left(I_{p}, A\right)\left(i \in\{1,2\}, j \in\left\{1, \ldots, l_{p}\right\}\right)$. Arrange all cubes $G_{p, j}, p \in \mathbb{N}, j \in\left\{1, \ldots, l_{p}\right\}$, in a sequence $\left\{G_{n}: n \in \mathbb{N}\right\}$. Then clearly (i) and (ii) are satisfied.

To prove (iii), fix an $i \in\{1,2\}$ and an $x \in A$. Let $l \in \mathbb{N}$. Since $h_{i}$ is quasi-continuous at $x$, there is an open set $H$ such that $\operatorname{diam}(\{x\} \cup H)<1 / l$ and $\omega\left(h_{i},\{x\} \cup H\right)<1 / l$. Then by (i), there exists an $n_{l} \in \mathbb{N}$ such that $\operatorname{int} G_{n_{l}} \cap H \neq \emptyset$, whence $\varrho\left(G_{n_{l}}, A\right)<1 / l$ and

$$
\omega\left(h_{i},\{x\} \cup G_{n_{l}}\right) \leq \omega\left(h_{i}, G_{n_{l}}\right)+\omega\left(h_{i},\{x\} \cup H\right) \leq 2 / l .
$$

Hence $x \in \operatorname{cl} \bigcup_{l \in \mathbb{N}} \operatorname{int} G_{n_{l}}$ and $h_{i} \mid\left(\{x\} \cup \bigcup_{l \in \mathbb{N}} \operatorname{int} G_{n_{l}}\right)$ is continuous at $x$, which completes the proof.

Lemma 3.3 Suppose $I$ is an interval, $L, \varepsilon>0$ and $h: \mathbb{R}^{k} \rightarrow \mathbb{R}$ is continuous at each point of a non-empty perfect set $K \subset \operatorname{int} I$. Then there is a continuous function $\alpha$ such that $\|\alpha\| \leq L+\left\|h \cdot \chi_{K}\right\|, \alpha=0$ outside of $I, \int_{I}|\alpha|<\varepsilon$, and

$$
(h+\alpha)(K) \cap(h-\alpha)(K) \supset[-L, L] .
$$

Proof. Clearly we may assume that $|I|<\varepsilon /\left(L+\left\|h \cdot \chi_{K}\right\|\right)$. Find disjoint non-empty perfect sets $K_{1}, K_{2} \subset K$ and continuous functions $\varphi_{1}, \varphi_{2}$ such that $\varphi_{i}\left(K_{i}\right)=[-L, L](i \in\{1,2\})$. Then use the Tietze extension theorem (see [4, Theorem 2.1.7]) to construct a continuous function $\alpha$ such that $\alpha=0$ outside of $I,\|\alpha\| \leq L+\left\|h \cdot \chi_{K}\right\|$ and $\alpha=(-1)^{i} \cdot h+\varphi_{i}$ on $K_{i}(i \in\{1,2\})$. It is obvious that (1) holds.

\section{Sums of Quasi-Continuous Functions}

Lemma 4.1 Suppose $\bar{g}, \bar{\alpha}$ and $g$ are functions which are continuous on a dense open set $G$, and $\bar{g} / 2+\bar{\alpha}, \bar{g} / 2-\bar{\alpha}$ are quasi-continuous on $A=\mathbb{R}^{k} \backslash G$. Then we can find an o-Lebesgue function $\alpha$ which is continuous on $G$ and such that 
(i) $\alpha=0$ on $A$,

(ii) $\|\alpha\| \leq\|g-\bar{g}\|$,

(iii) functions $g / 2+\bar{\alpha}+\alpha$ and $g / 2-\bar{\alpha}-\alpha$ are quasi-continuous on $A$.

Proof. First find a family of non-overlapping cubes $\left\{G_{n}: n \in \mathbb{N}\right\}$ according to Lemma 3.2 (with $h_{i}=\bar{g} / 2+(-1)^{i} \cdot \bar{\alpha}, i \in\{1,2\}$ ). Then for each $n \in \mathbb{N}$ apply Lemma 3.3 with $I=G_{n}, L=L_{n}=\min \{n,\|g-\bar{g}\| / 2\}, \varepsilon=2^{-n}$ and $h=(g-\bar{g}) / 2$, and find a continuous function $\alpha_{n}$ such that $\left\|\alpha_{n}\right\| \leq\|g-\bar{g}\|$, $\alpha_{n}=0$ outside of $G_{n}, \int_{G_{n}}\left|\alpha_{n}\right|<2^{-n} \cdot\left|G_{n}\right|$ and for $i \in\{1,2\}$

$$
\left(\frac{g-\bar{g}}{2}+(-1)^{i} \cdot \alpha_{n}\right)\left(\operatorname{int} G_{n}\right) \supset\left[-L_{n}, L_{n}\right] .
$$

Define $\alpha=\sum_{n \in \mathbb{N}} \alpha_{n}$. By Theorem 2.2, each $x \in A$ is an $o$-Lebesgue point of $\alpha$ and since by condition (i) of Lemma 3.2, $\alpha$ is continuous on $G, \alpha$ is an $o$-Lebesgue function. Clearly conditions (i) and (ii) are satisfied.

To prove (iii), take an $i \in\{1,2\}$ and an $x \in A$. By condition (iii) of Lemma 3.2 , there is a subfamily $\left\{G_{n_{l}}: l \in \mathbb{N}\right\}$ such that $x \in \operatorname{cl} \bigcup_{l \in \mathbb{N}}$ int $G_{n_{l}}$ and $\left(\bar{g} / 2+(-1)^{i} \cdot \bar{\alpha}\right) \mid\left(\{x\} \cup \bigcup_{l \in \mathbb{N}}\right.$ int $\left.G_{n_{l}}\right)$ is continuous at $x$. Put

$$
H=\bigcup_{l \in \mathbb{N}}\left(\operatorname{int} G_{n_{l}} \cap\left(\frac{g-\bar{g}}{2}+(-1)^{i} \cdot \alpha\right)^{-1}((c-1 / l, c+1 / l))\right),
$$

where $c=(g(x)-\bar{g}(x)) / 2$. Then $H$ is open and by (2), $x \in \mathrm{cl} H$. It is clear that $\left(g / 2+(-1)^{i} \cdot \bar{\alpha}+(-1)^{i} \cdot \alpha\right) \mid(\{x\} \cup H)$ is continuous at $x$, so (iii) holds. $\square$

Theorem 4.2 Given a cliquish function $f$ and an $\eta>0$ we can find an $o$-Lebesgue function $\alpha$ such that the functions $f / 2+\alpha$ and $f / 2-\alpha$ are quasicontinuous, $D(\alpha) \subset D(f)$ and $\|\alpha\| \leq\|f\|+\eta$.

Proof. First use Lemma 3.1 with $G=\left\{x \in \mathbb{R}^{k}: \omega(f, x)<\eta \cdot 2^{-n}\right\}$ to find a sequence of functions $\left\{f_{n}: n \in \mathbb{N}\right\}$ such that for each $n \in \mathbb{N}$ : the set $A_{n}=\operatorname{cl} D\left(f_{n}\right)$ is contained in $D(f), f=f_{n}$ on $A_{n}$ and $\left|f-f_{n}\right| \leq \eta \cdot 2^{-n-1}$ on $\mathbb{R}^{k} \backslash A_{n}$.

Set $f_{0}=0$. For each $n \in \mathbb{N}$ use Lemma 4.1 with $\bar{g}=f_{n-1}, \bar{\alpha}=\sum_{j<n} \alpha_{j}$, $g=f_{n}$ and $G=\mathbb{R}^{k} \backslash \bigcup_{j \leq n} A_{j}$, to find an $o$-Lebesgue function $\alpha_{n}$ which is continuous outside of $\bigcup_{j \leq n} A_{j}, \alpha_{n}=0$ on $\bigcup_{j \leq n} A_{j},\left\|\alpha_{n}\right\| \leq\left\|f_{n}-f_{n-1}\right\|$ and such that for $i \in\{1,2\}$ the function $f_{n} / 2+(-1)^{i} \cdot \sum_{j \leq n} \alpha_{j}$ is quasi-continuous.

Set $\alpha=\sum_{n=1}^{\infty} \alpha_{n}$. Note that this series is uniformly convergent (so $\alpha$ is an $o$-Lebesgue function) and

$$
\|\alpha\| \leq \sum_{n=1}^{\infty}\left\|f_{n}-f_{n-1}\right\| \leq\|f\|+\eta / 4+3 \cdot \sum_{n=2}^{\infty} \eta \cdot 2^{-n-1}=\|f\|+\eta .
$$


Clearly $D(\alpha) \subset D(f)$.

Fix an $i \in\{1,2\}$. Observe that $f / 2+(-1)^{i} \cdot \alpha$ is the uniform limit of the sequence $\left\{f_{n} / 2+(-1)^{i} \cdot \sum_{j \leq n} \alpha_{j}: n \in \mathbb{N}\right\}$ (which consists of quasi-continuous functions), so this function is quasi-continuous.

\section{Products of Quasi-Continuous Functions}

We start with a lemma.

Lemma 5.1 Assume that a function $\widetilde{g}$ is cliquish and positive on an interval I. Then we can find quasi-continuous functions $\widetilde{h}^{(1)}, \widetilde{h}^{(2)}$ such that

(a) $\widetilde{g}=\widetilde{h}^{(1)} \cdot \widetilde{h}^{(2)}$ on $I$,

(b) for each $x \in$ fr $I$, each $i \in\{1,2\}$ and each $s \geq 0$ there is a sequence $x_{1}, x_{2}, \cdots \in \operatorname{int} I \backslash D\left(\widetilde{h}^{(i)}\right)$ such that $x_{n} \rightarrow x$ and $\widetilde{h}^{(i)}\left(x_{n}\right) \rightarrow s$.

Proof. Define the function $f$ by

$$
f(x)= \begin{cases}\ln \widetilde{g}(x) & \text { if } x \in I, \\ 0 & \text { otherwise }\end{cases}
$$

and apply Theorem 4.2 to find quasi-continuous functions $h^{(1)}, h^{(2)}$ such that $f=h^{(1)}+h^{(2)}$ on $\mathbb{R}^{k}$. Use Lemma 2.1 with $A=\mathbb{R}^{k} \backslash \operatorname{int} I$ to find a family $\mathcal{I}=\left\{I_{n}: n \in \mathbb{N}\right\}$ of non-overlapping cubes satisfying its requirements.

For each $n \in \mathbb{N}$ use Lemma 3.3 (twice) to find a continuous function $\alpha_{n}$ such that $\alpha_{n}=0$ on fr $I_{n}$ and for $i \in\{1,2\}$

$$
\left(h^{(i)}+(-1)^{i} \cdot \alpha_{n}\right)\left(\operatorname{int} I_{n} \backslash D(\widetilde{g})\right) \supset[-n, n] .
$$

Define for $i \in\{1,2\}$ function $\widetilde{h}^{(i)}$ by

$$
\widetilde{h}^{(i)}(x)= \begin{cases}\exp \left(h^{(i)}(x)+(-1)^{i} \cdot \alpha_{n}(x)\right) & \text { if } x \in I_{n}^{\circ}, n \in \mathbb{N} \\ \exp \left(h^{(i)}(x)\right) & \text { otherwise. }\end{cases}
$$

Then clearly (a) holds. To prove (b) fix an $x \in$ fr $I$, an $i \in\{1,2\}$, and an $s \geq 0$.

First suppose $s>0$. Let $n \in \mathbb{N}$. Take an arbitrary $y_{n} \in \operatorname{int} I$ such that $\varrho\left(x, y_{n}\right)<\min \left\{1 / n, \varrho\left(x, \bigcup_{l<|\ln s|} I_{l}\right)\right\}$. Then $y_{n} \in I_{l_{n}}$ for some $l_{n} \geq|\ln s|$ and so by (3), there is an $x_{n} \in I_{l_{n}} \backslash D(\widetilde{g})$ such that $\widetilde{h}^{(i)}\left(x_{n}\right)=s$. Since $\varrho\left(x, x_{n}\right) \leq 2 / n$; so $x_{n} \rightarrow x$.

The case $s=0$ immediately follows from the fact that for every $s>0$ we are done.

Finally note that by (b), the functions $\widetilde{h}^{(1)}$ and $\widetilde{h}^{(2)}$ are quasi-continuous on fr $I$. Quasi-continuity outside of $\operatorname{fr} I$ is evident. 
Theorem 5.2 For each function $g$ the following conditions are equivalent:

(a) There is an $l \in \mathbb{N}$ and quasi-continuous functions $h^{(1)}, \ldots, h^{(l)}$ such that $g=h^{(1)} \ldots \ldots h^{(l)}$.

(b) There are quasi-continuous functions $h^{(1)}$ and $h^{(2)}$ with $g=h^{(1)} \cdot h^{(2)}$.

(c) Function $g$ is cliquish and it satisfies condition $(\star)$ of Theorem 1.1 .

Proof. The proof of the implication $(\mathrm{a}) \Rightarrow(\mathrm{c})$ is a repetition of the argument used in [11], while the implication (b) $\Rightarrow$ (a) is obvious; so we need only to prove (c) $\Rightarrow$ (b). Set $G_{0}=\operatorname{int}\left\{x \in \mathbb{R}^{k}: g(x)=0\right\}, G_{1}=\operatorname{int}\left\{x \in \mathbb{R}^{k}: g(x)<0\right\}$, and $G_{2}=\operatorname{int}\left\{x \in \mathbb{R}^{k}: g(x)>0\right\}$. For $p \in\{0,1,2\}$ apply Lemma 2.1 with $A=\mathbb{R}^{k} \backslash G_{p}$ to find a family $\left\{I_{p, n}: n \in \mathbb{N}\right\}$ of non-overlapping cubes satisfying its conditions. Fix an $n \in \mathbb{N}$.

Find continuous functions $h_{0, n}^{(1)}$ and $h_{0, n}^{(2)}$ such that $h_{0, n}^{(1)} \cdot h_{0, n}^{(2)}=0$ on $\mathbb{R}^{k}$, $h_{0, n}^{(1)}=h_{0, n}^{(2)}=0$ on fr $I_{0, n}$, and

$$
h_{0, n}^{(1)}\left(\operatorname{int} I_{0, n}\right) \cap h_{0, n}^{(2)}\left(\operatorname{int} I_{0, n}\right) \supset[-n, n] .
$$

For $p \in\{1,2\}$ do the following. Find non-overlapping intervals $I_{p, n, 1}$ and $I_{p, n, 2}$ such that $I_{p, n}=I_{p, n, 1} \cup I_{p, n, 2}$. For $j \in\{1,2\}$ use Lemma 5.1 with $\widetilde{g}=|g|$ and $I=I_{p, n, j}$ to get quasi-continuous functions $\widetilde{h}_{p, n, j}^{(1)}, \widetilde{h}_{p, n, j}^{(2)}$ satisfying its conditions.

Now for $i \in\{1,2\}$ define the function $h^{(i)}$ by

$$
h^{(i)}(x)= \begin{cases}h_{0, n}^{(i)}(x) & \text { if } x \in I_{0, n}^{\circ}, n \in \mathbb{N}, \\ (-1)^{j} \cdot[\operatorname{sgn} f(x)]^{i} \cdot \widetilde{h}_{p, n, j}^{(i)}(x) & \text { if } x \in I_{p, n, j}^{\circ}, n \in \mathbb{N}, p, j \in\{1,2\}, \\ (2-i) \cdot(f(x)-1)+1 & \text { otherwise. }\end{cases}
$$

Then it is easy to prove that for each $x \notin G_{0} \cup G_{1} \cup G_{2}, i \in\{1,2\}$ and $s \in \mathbb{R}$ there is a sequence $x_{1}, x_{2}, \cdots \in \mathbb{R}^{k} \backslash D\left(h^{(i)}\right)$ such that $x_{n} \rightarrow x$ and $h^{(i)}\left(x_{n}\right) \rightarrow s$. (Note that $G_{0} \cup G_{1} \cup G_{2}$ is dense in $\mathbb{R}^{k}$, by $(\star)$.) So the functions $h^{(1)}$ and $h^{(2)}$ are quasi-continuous.

\section{Remarks}

Remark 6.1 If $f$ is a function which is discontinuous on a nowhere dense set, then by Lemma 4.1, we can find quasi-continuous functions $h_{1}, h_{2}$ such that $\left\|h_{i}\right\| \leq 3\|f\| / 2$ for $i \in\{1,2\}$ and $h_{1}+h_{2}=f$. On the other hand, it is easy to show that $\left\|h_{1}\right\|+\left\|h_{2}\right\| \geq 3$ whenever quasi-continuous functions $h_{1}$ and $h_{2}$ are such that $h_{1}+h_{2}=2 \chi_{\{0\}}-1$. However, I do not know whether the following assertion is true: 
Whenever $f$ is a bounded cliquish function we can find quasi-continuous functions $h_{1}, h_{2}$ such that $\left\|h_{1}\right\|+\left\|h_{2}\right\| \leq 3\|f\|$ and $h_{1}+$ $h_{2}=f$.

Remark 6.2 Since each o-Lebesgue function belongs to the first class of Baire (cf. [9]), the following assertion holds:

Let $\mathcal{A}$ be the family of all measurable functions or Baire class $\alpha$. Then for each cliquish function $f \in \mathcal{A}$ and each $\eta>0$ we can find quasi-continuous functions $h_{1}, h_{2} \in \mathcal{A}$ such that $f=h_{1}+h_{2}$, $D\left(h_{1}\right) \cup D\left(h_{2}\right) \subset D(f)$ and $\left\|h_{i}\right\| \leq 3\|f\| / 2+\eta$ for $i \in\{1,2\}$.

Though o-Lebesgue functions need not be Baire one star functions ( $f$ is a Baire one star function if for each non-empty perfect set $P$ there is an interval $I$ with $P \cap \operatorname{int} I \neq \emptyset$ such that $f \mid(P \cap \operatorname{int} I)$ is continuous $)$, the above assertion remains true if $\mathcal{A}$ is the family of all Baire one star functions, by Lemma 4.1. (Cf. also [6].)

Remark 6.3 Suppose that a system of functions $\mathcal{A}$ satisfies the following postulates:

- $\mathcal{A}$ is a vector space containing all o-Lebesgue functions,

- for any function $f$ if there are a closed set $A$, functions $h, h_{1}, h_{2}, \cdots \in \mathcal{A}$ and a family $\left\{I_{n}: n \in \mathbb{N}\right\}$ of non-overlapping cubes satisfying requirements of Lemma 2.1 such that $f=h$ on $A$ and $f=h_{n}$ on $I_{n}^{\circ}$ for each $n \in \mathbb{N}$ then $f \in \mathcal{A}$,

- $\mathcal{A}$ is closed with respect to outer compositions with continuous functions.

Then in the condition (b) of Theorem 5.2 we can moreover require that the functions $h^{(1)}, h^{(2)} \in \mathcal{A}$ provided that $g \in \mathcal{A}$. (The proof remains unchanged.)

Remark 6.4 It is easy to prove that each Baire one star function is cliquish and satisfies condition $(\star)$ of Theorem 1.1. So the following assertion holds.

Each Baire one star function can be factored into a product of two Baire one star quasi-continuous functions.

(In the proof of the analogue of Lemma 5.1 we use Lemma 4.1 in place of Theorem 4.2.) On the other hand, not every Darboux Baire one star function is a product of Darboux Baire one star functions [6]. 


\section{References}

[1] J. Borsík, Sums of quasicontinuous functions, Math. Bohemica, 118 no. 3 (1993), 313-319.

[2] J. Borsík, Products of simply continuous and quasicontinuous functions, to appear in Math. Slovaca.

[3] J. Borsík and J. Doboš, A note on real cliquish functions, Real Anal. Exchange, 18 no. 1 (1992-93), 139-145.

[4] R. Engelking, General topology, Monografie Matematyczne, vol. 32, PWN, Warszawa, 1976.

[5] Z. Grande, Sur le fonctions cliquish, Časopis Pěst. Mat., 110 (1985), $225-236$.

[6] Z. Grande, On the sums and products of Darboux Baire* 1 functions, Real Anal. Exchange, 18 no. 1 (1992-93), 237-240.

[7] Z. Grande, On Borsik's problem concerning quasiuniform limits of Darboux quasicontinuous functions, Math. Slovaca, 44 (1994), 297-301.

[8] S. Kempisty, Sur les fonctions quasicontinues, Fund. Math., 19 (1932), 184-197.

[9] J. Lukeš, J. Malý, and L. Zajíček, Fine topology methods in real analysis and potential theory, Lect. Notes in Math., vol. 1189, Springer Verlag, 1986.

[10] A. Maliszewski, Integration of derivatives of additive interval functions, submitted.

[11] T. Natkaniec, Products of quasi-continuous functions, Math. Slovaca, 40 no. 4 (1990), 401-405.

[12] T. Neubrunn, Quasi-continuity, Real Anal. Exchange, 14 no. 2 (1988-89), 259-306.

[13] E. Strońska, L'espace linéaire des fonctions cliquées sur $\mathbb{R}^{n}$ est généré par les fonctions quasi-continues, Math. Slovaca, 39 (1989), 155-164.

[14] E. Strońska, On the group generated by quasi continuous functions, Real Anal. Exchange, 17 no. 2 (1991-92), 577-589. 